\title{
FFCASP: A Massively Parallel Crystal Structure Prediction Algorithm
}

Samet Demir ${ }^{1,2}$ and Adem Tekin ${ }^{1,2}$

1) Informatics Institute, Istanbul Technical University, 34469 Maslak, Istanbul, Turkey

2) TÜBİTAK Research Institute for Fundamental Sciences, 41470 Gebze, Kocaeli, Turkey 


\section{LIST OF FIGURES}

S1 Relative total energies for low energy cytosine crystals obtained from different dispersion-inclusive DFT methods. . . . . . . . . . . . . . . . . . 2

S2 Crystal structure of phonon stable monoclinic structure $\mathrm{P} 2_{1} / \mathrm{c}$ of cytosine... 2

S3 Crystal structure of phonon stable tetragonal structure İ $2 \mathrm{~d}$ of cytosine. ... . 3

S4 Crystal structure of phonon stable orthorhombic structure Fdd2 of cytosine. . 4

S5 Relative total energies for low energy coumarin crystals obtained from differ-

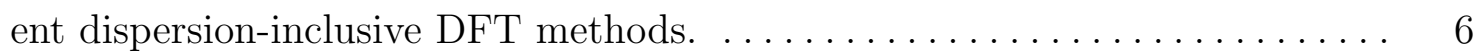

S6 Crystal structure of phonon stable triclinic structure P1 of coumarin. ...... 6

S7 Relative total energies for low energy pyrazinamide crystals obtained from different dispersion-inclusive DFT methods. . . . . . . . . . . . . . 8

S8 Crystal structure of phonon stable monoclinic structure P2_1 of pyrazinamide. 8

S9 Crystal structure of phonon stable monoclinic structure P2_1/c of pyrazinamide. 9



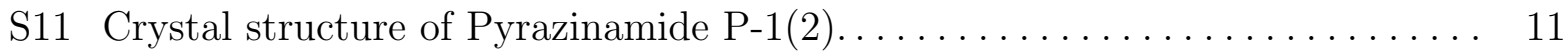

S12 Coumarin dimers which used as identifier of dendrogram clusters ......... 13

S13 Pyrazinamide dimers which used as identifier of dendrogram clusters . . . . . 14

S14 Space group distrubution of DFT relaxed cytosine structures. (P1 and P-1

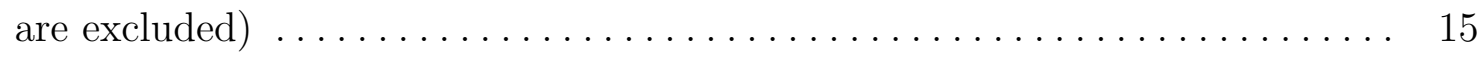

S15 Space group distrubution of DFT relaxed coumarin structures. (P1 and P-1

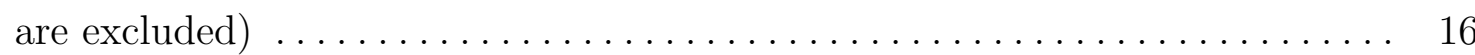

S16 Space group distrubution of DFT relaxed pyrazinamide structures. (P1 and



\section{LIST OF TABLES}

S1 Crystallographic data the monoclinic structure $\mathrm{P} 2{ }_{1} / \mathrm{c}$ of cytosine. ....... 3

S2 Crystallographic data the tetragonal structure I $\overline{4} 2 \mathrm{~d}$ of cytosine......... 4

S3 Crystallographic data the orthorhombic structure Fdd2 of cytosine. ....... 5

S4 Crystallographic data the triclinic structure P1 of coumarin............. 7

S5 Crystallographic data the monoclinic structure P2_1 of pyrazinamide...... 9 
S6 Crystallographic data the monoclinic structure P2_1/c of pyrazinamide. . . . 10

S7 Crystallographic data the triclinic structure P-1(1) of pyrazinamide....... 11

S8 Crystallographic data the triclinic structure P-1(2) of pyrazinamide....... 12 


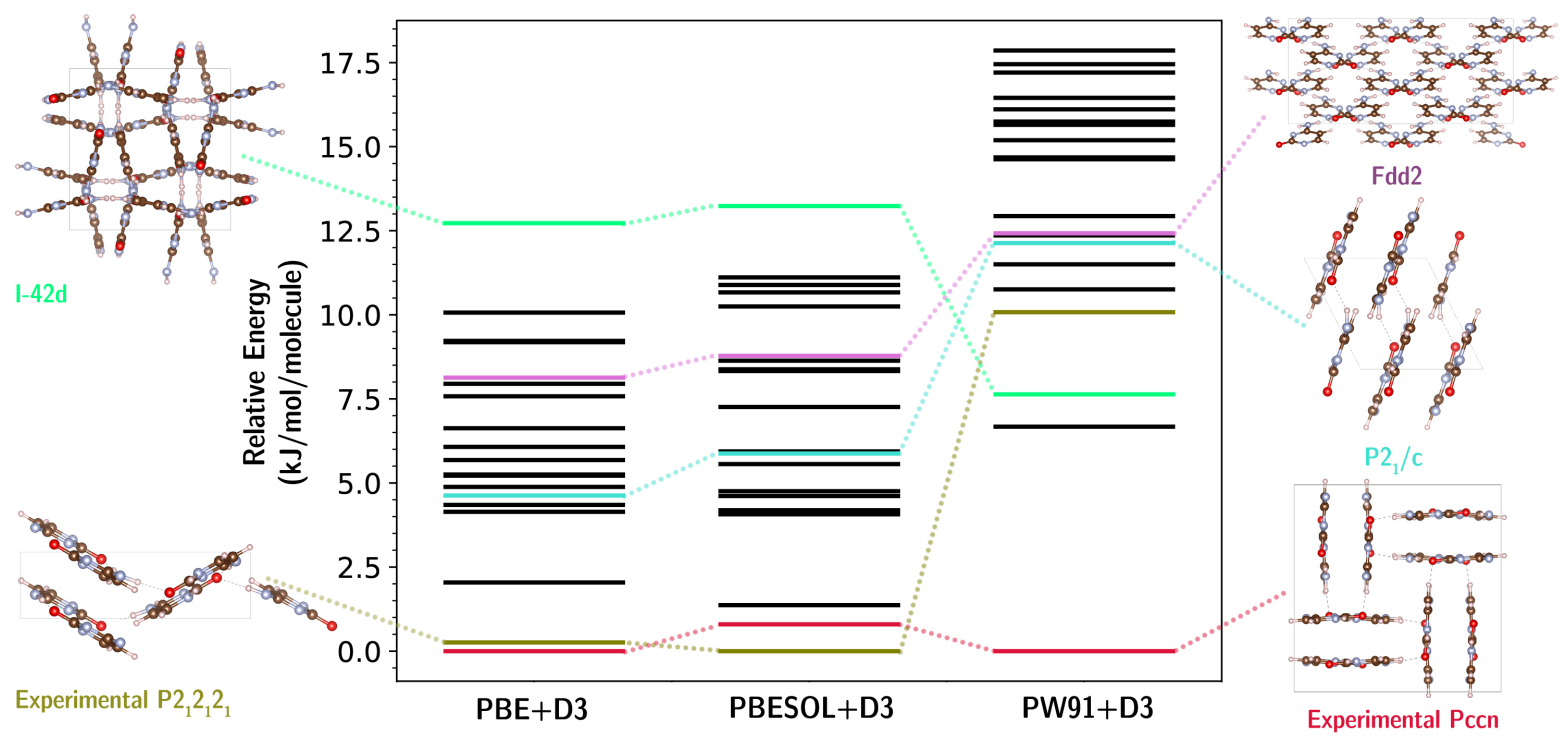

FIG. S1. Relative total energies for low energy cytosine crystals obtained from different dispersioninclusive DFT methods.
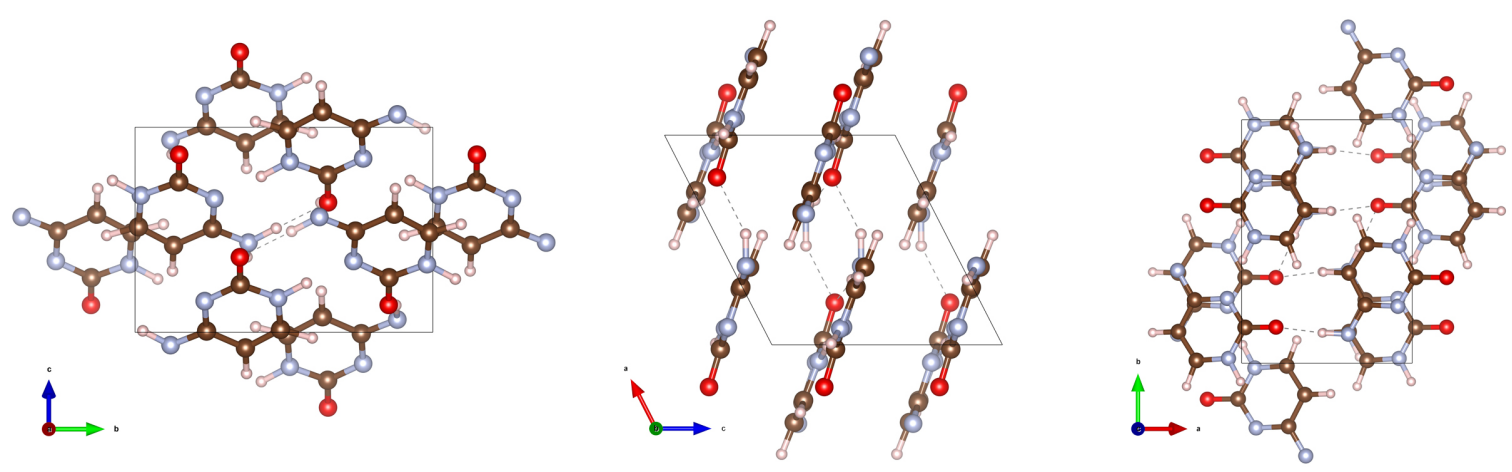

FIG. S2. Crystal structure of phonon stable monoclinic structure P2 $2_{1} /$ c of cytosine. 


\begin{tabular}{|c|c|c|c|c|}
\hline Space group name & $\mathbf{a}[\AA]$ & $\mathbf{b}[\AA]$ & $\mathbf{c}[\AA]$ & Cell volume $\left[\AA^{3}\right]$ \\
\hline \multirow[t]{3}{*}{$\mathbf{P 2}_{1} / \mathrm{c}(\# \mathbf{1 4})$} & 7.5251 & 9.5279 & 7.3712 & 470.2767 \\
\hline & $\alpha$ & $\beta$ & $\gamma$ & \\
\hline & 90.0000 & 117.1506 & 90.0000 & \\
\hline Species & $\mathbf{x}$ & $y$ & $\mathbf{z}$ & Site \\
\hline $\mathrm{H}$ & 0.0064 & 0.0757 & 0.2599 & $4 \mathrm{e}$ \\
\hline $\mathrm{H}$ & 0.3097 & 0.5260 & 0.4927 & $4 \mathrm{e}$ \\
\hline $\mathrm{H}$ & 0.3229 & 0.0949 & 0.5270 & $4 \mathrm{e}$ \\
\hline $\mathrm{H}$ & 0.4721 & 0.1243 & 0.8678 & $4 \mathrm{e}$ \\
\hline $\mathrm{H}$ & 0.4787 & 0.1255 & 0.2996 & $4 \mathrm{e}$ \\
\hline $\mathrm{C}$ & 0.0206 & 0.1468 & 0.7332 & $4 \mathrm{e}$ \\
\hline $\mathrm{C}$ & 0.2650 & 0.5112 & 0.9856 & $4 \mathrm{e}$ \\
\hline $\mathrm{C}$ & 0.2752 & 0.7401 & 0.4937 & $4 \mathrm{e}$ \\
\hline $\mathrm{C}$ & 0.3705 & 0.6293 & 0.0759 & $4 \mathrm{e}$ \\
\hline $\mathrm{N}$ & 0.0771 & 0.5195 & 0.8324 & $4 \mathrm{e}$ \\
\hline $\mathrm{N}$ & 0.0845 & 0.7338 & 0.3478 & $4 e$ \\
\hline $\mathrm{N}$ & 0.3756 & 0.6190 & 0.5623 & $4 \mathrm{e}$ \\
\hline $\mathrm{O}$ & 0.2010 & 0.1481 & 0.8663 & $4 e$ \\
\hline
\end{tabular}

TABLE S1. Crystallographic data the monoclinic structure $\mathrm{P} 2_{1} / \mathrm{c}$ of cytosine.


FIG. S3. Crystal structure of phonon stable tetragonal structure İ̄2d of cytosine. 


\begin{tabular}{|c|c|c|c|c|}
\hline Space group name & $\mathbf{a}[\AA]$ & $\mathbf{b}[\AA]$ & $\mathbf{c}[\AA]$ & Cell volume $\left[\AA^{3}\right]$ \\
\hline \multirow{3}{*}{$\mathbf{I} \overline{4} \mathbf{2 d}(\# \mathbf{1 2 2})$} & 10.0917 & 10.0917 & 21.6393 & 2203.8168 \\
\hline & $\alpha$ & $\beta$ & $\gamma$ & \\
\hline & 90.0000 & 90.0000 & 90.0000 & \\
\hline Species & $\mathbf{x}$ & $\mathrm{y}$ & $\mathbf{z}$ & Site \\
\hline $\mathrm{H}$ & 0.0853 & 0.2038 & 0.9954 & $16 \mathrm{e}$ \\
\hline $\mathrm{H}$ & 0.0894 & 0.8541 & 0.6947 & $16 \mathrm{e}$ \\
\hline $\mathrm{H}$ & 0.1053 & 0.1804 & 0.0772 & $16 \mathrm{e}$ \\
\hline $\mathrm{H}$ & 0.1536 & 0.3071 & 0.5359 & $16 \mathrm{e}$ \\
\hline $\mathrm{H}$ & 0.1975 & 0.7302 & 0.0685 & $16 \mathrm{e}$ \\
\hline $\mathrm{C}$ & 0.0473 & 0.1769 & 0.8605 & $16 \mathrm{e}$ \\
\hline $\mathrm{C}$ & 0.0492 & 0.8433 & 0.7410 & $16 \mathrm{e}$ \\
\hline $\mathrm{C}$ & 0.0812 & 0.1823 & 0.7510 & $16 \mathrm{e}$ \\
\hline $\mathrm{C}$ & 0.1314 & 0.6377 & 0.4556 & $16 \mathrm{e}$ \\
\hline $\mathrm{N}$ & 0.0824 & 0.8483 & 0.8521 & $16 \mathrm{e}$ \\
\hline $\mathrm{N}$ & 0.1046 & 0.2410 & 0.0385 & $16 \mathrm{e}$ \\
\hline $\mathrm{N}$ & 0.1289 & 0.1924 & 0.8094 & $16 \mathrm{e}$ \\
\hline $\mathrm{O}$ & 0.0991 & 0.1861 & 0.9135 & $16 \mathrm{e}$ \\
\hline
\end{tabular}

TABLE S2. Crystallographic data the tetragonal structure I⿳亠厶冋2d of cytosine.
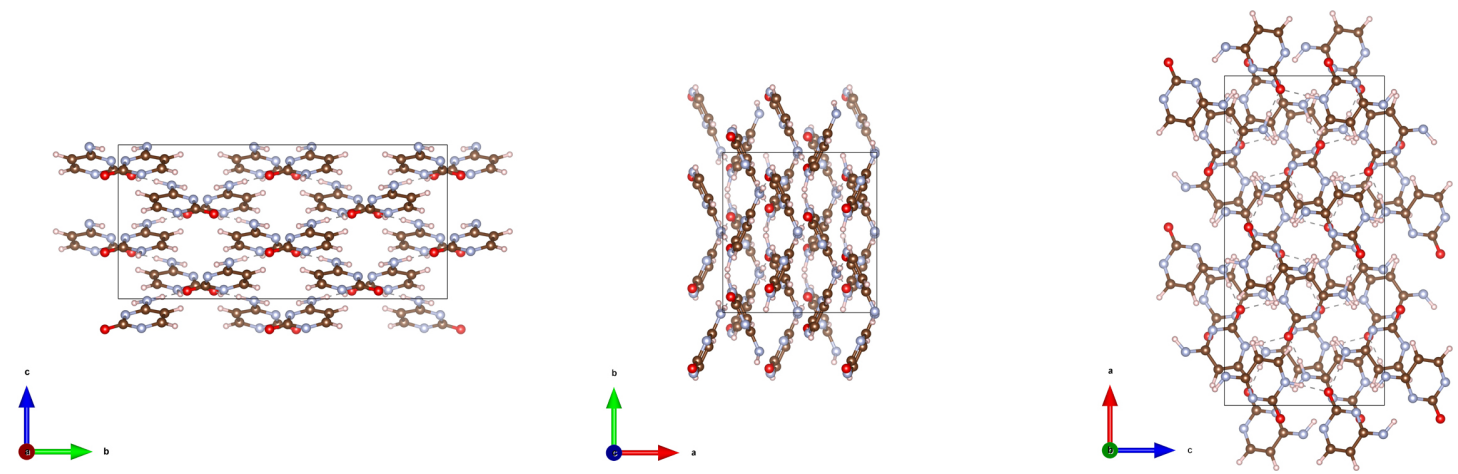

FIG. S4. Crystal structure of phonon stable orthorhombic structure Fdd2 of cytosine. 


\begin{tabular}{|c|c|c|c|c|}
\hline Space group name & $\mathbf{a}[\AA]$ & $\mathbf{b}[\AA]$ & $\mathbf{c}[\AA]$ & Cell volume $\left[\AA^{3}\right]$ \\
\hline \multirow[t]{3}{*}{ Fdd2 $(\# 43)$} & 9.8390 & 20.2426 & 9.4545 & 1883.0410 \\
\hline & $\alpha$ & $\beta$ & $\gamma$ & \\
\hline & 90.0000 & 90.0000 & 90.0000 & \\
\hline Species & $\mathrm{x}$ & $\mathbf{y}$ & $\mathbf{z}$ & Site \\
\hline $\mathrm{H}$ & 0.0208 & 0.4398 & 0.7828 & $16 \mathrm{~b}$ \\
\hline $\mathrm{H}$ & 0.0270 & 0.3667 & 0.5097 & $16 \mathrm{~b}$ \\
\hline $\mathrm{H}$ & 0.0580 & 0.4509 & 0.4700 & $16 \mathrm{~b}$ \\
\hline $\mathrm{H}$ & 0.0729 & 0.4377 & 0.1853 & $16 \mathrm{~b}$ \\
\hline $\mathrm{H}$ & 0.0932 & 0.3298 & 0.8236 & $16 \mathrm{~b}$ \\
\hline $\mathrm{C}$ & 0.0366 & 0.3892 & 0.1557 & $16 \mathrm{~b}$ \\
\hline $\mathrm{C}$ & 0.0500 & 0.2359 & 0.5855 & $16 \mathrm{~b}$ \\
\hline $\mathrm{C}$ & 0.0871 & 0.1197 & 0.5968 & $16 \mathrm{~b}$ \\
\hline $\mathrm{C}$ & 0.1178 & 0.3319 & 0.1786 & $16 \mathrm{~b}$ \\
\hline $\mathrm{N}$ & 0.0090 & 0.0872 & 0.9843 & $16 \mathrm{~b}$ \\
\hline $\mathrm{N}$ & 0.0733 & 0.2713 & 0.1436 & $16 \mathrm{~b}$ \\
\hline $\mathrm{N}$ & 0.1207 & 0.4309 & 0.8106 & $16 \mathrm{~b}$ \\
\hline $\mathrm{O}$ & 0.0982 & 0.2913 & 0.5527 & $16 \mathrm{~b}$ \\
\hline
\end{tabular}

TABLE S3. Crystallographic data the orthorhombic structure Fdd2 of cytosine. 


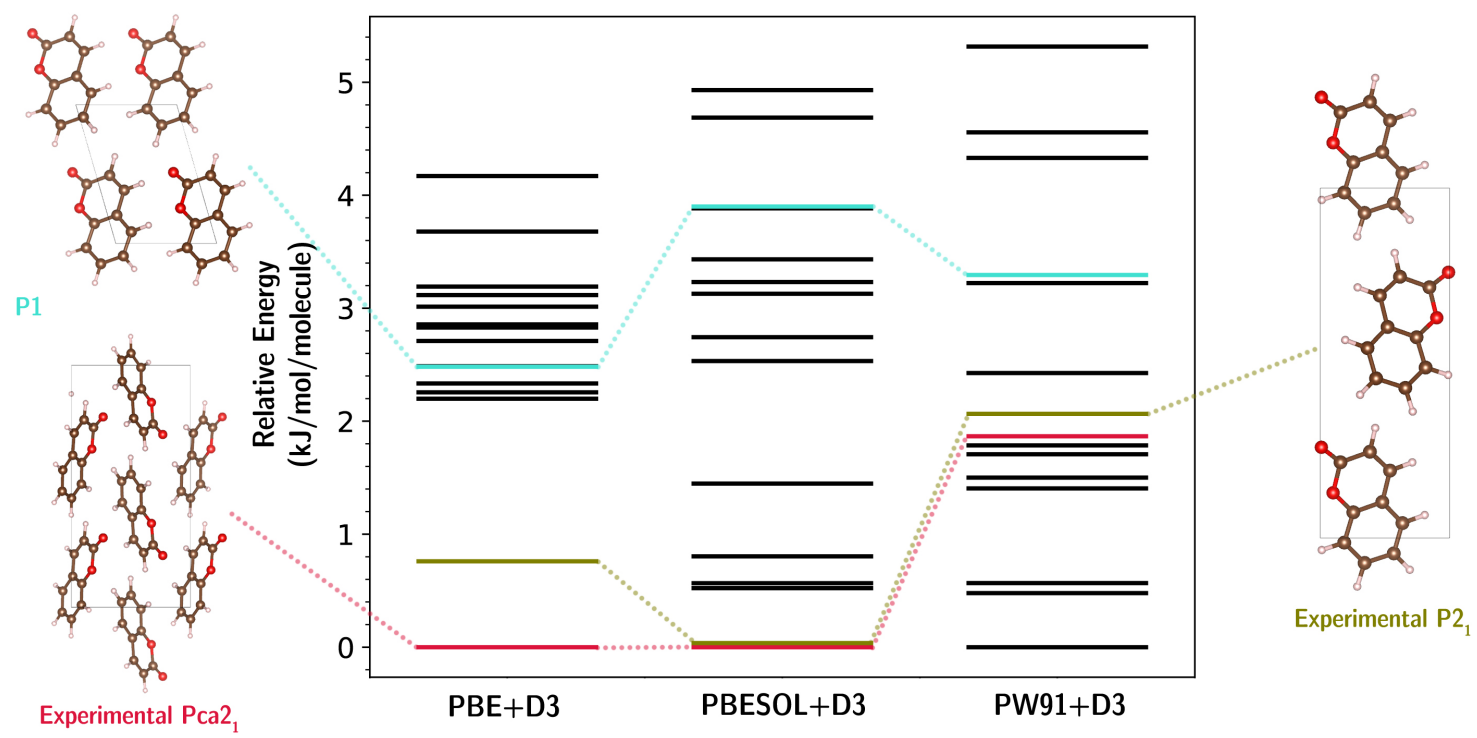

FIG. S5. Relative total energies for low energy coumarin crystals obtained from different dispersioninclusive DFT methods.
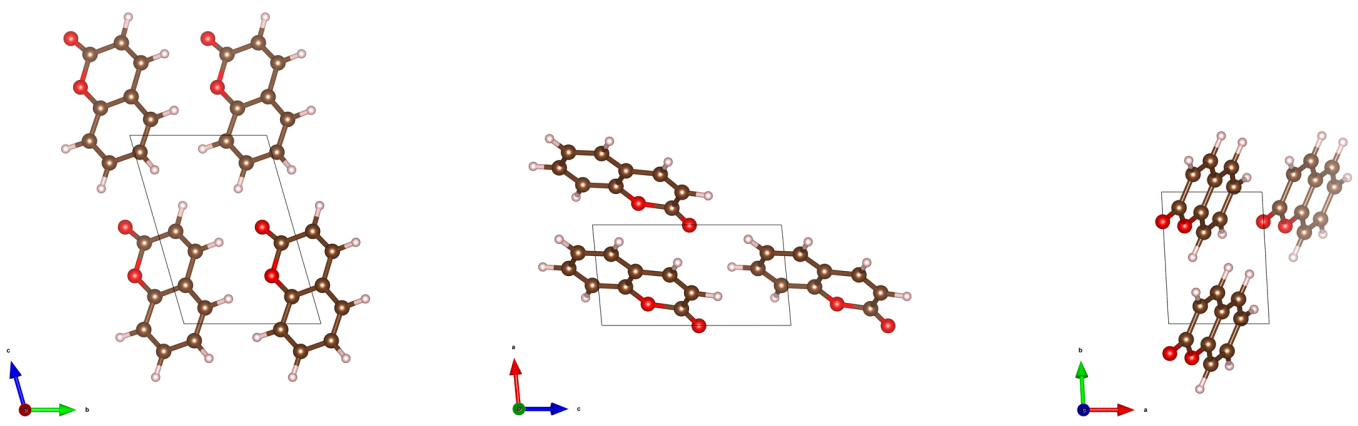

FIG. S6. Crystal structure of phonon stable triclinic structure P1 of coumarin. 


\begin{tabular}{|c|c|c|c|c|}
\hline Space group name & $\mathbf{a}[\AA]$ & $\mathbf{b}[\AA]$ & $\mathbf{c}[\AA]$ & Cell volume $\left[\AA^{3}\right]$ \\
\hline \multirow[t]{3}{*}{$\mathbf{P 1}(\# 1)$} & 4.0213 & 5.4566 & 7.8308 & 164.4554 \\
\hline & $\alpha$ & $\beta$ & $\gamma$ & \\
\hline & 105.8901 & 94.7559 & 91.6772 & \\
\hline Species & $\mathbf{x}$ & $\mathbf{y}$ & $\mathbf{z}$ & Site \\
\hline $\mathrm{H}$ & 0.2750 & 0.5040 & 0.9277 & $1 \mathrm{a}$ \\
\hline $\mathrm{H}$ & 0.2887 & 0.2387 & 0.6268 & $1 \mathrm{a}$ \\
\hline $\mathrm{H}$ & 0.5815 & 0.6805 & 0.7166 & $1 \mathrm{a}$ \\
\hline $\mathrm{H}$ & 0.6246 & 0.4259 & 0.4320 & $1 \mathrm{a}$ \\
\hline $\mathrm{H}$ & 0.8308 & 0.3769 & 0.1320 & $1 \mathrm{a}$ \\
\hline $\mathrm{H}$ & 0.8563 & 0.1092 & 0.8165 & $1 \mathrm{a}$ \\
\hline $\mathrm{C}$ & 0.1666 & 0.8804 & 0.4285 & $1 \mathrm{a}$ \\
\hline $\mathrm{C}$ & 0.3237 & 0.1338 & 0.4904 & $1 \mathrm{a}$ \\
\hline $\mathrm{C}$ & 0.3830 & 0.8439 & 0.1436 & $1 \mathrm{a}$ \\
\hline $\mathrm{C}$ & 0.3982 & 0.6940 & 0.9694 & $1 \mathrm{a}$ \\
\hline $\mathrm{C}$ & 0.5028 & 0.2350 & 0.3840 & $1 \mathrm{a}$ \\
\hline $\mathrm{C}$ & 0.5365 & 0.0916 & 0.2044 & $1 \mathrm{a}$ \\
\hline $\mathrm{C}$ & 0.5667 & 0.7934 & 0.8533 & $1 \mathrm{a}$ \\
\hline $\mathrm{C}$ & 0.7085 & 0.1866 & 0.0843 & $1 \mathrm{a}$ \\
\hline $\mathrm{C}$ & 0.7217 & 0.0393 & 0.9102 & $1 \mathrm{a}$ \\
\hline $\mathrm{O}$ & 0.2126 & 0.7414 & 0.2544 & $1 \mathrm{a}$ \\
\hline $\mathrm{O}$ & 0.9962 & 0.7742 & 0.5126 & $1 \mathrm{a}$ \\
\hline
\end{tabular}

TABLE S4. Crystallographic data the triclinic structure P1 of coumarin. 

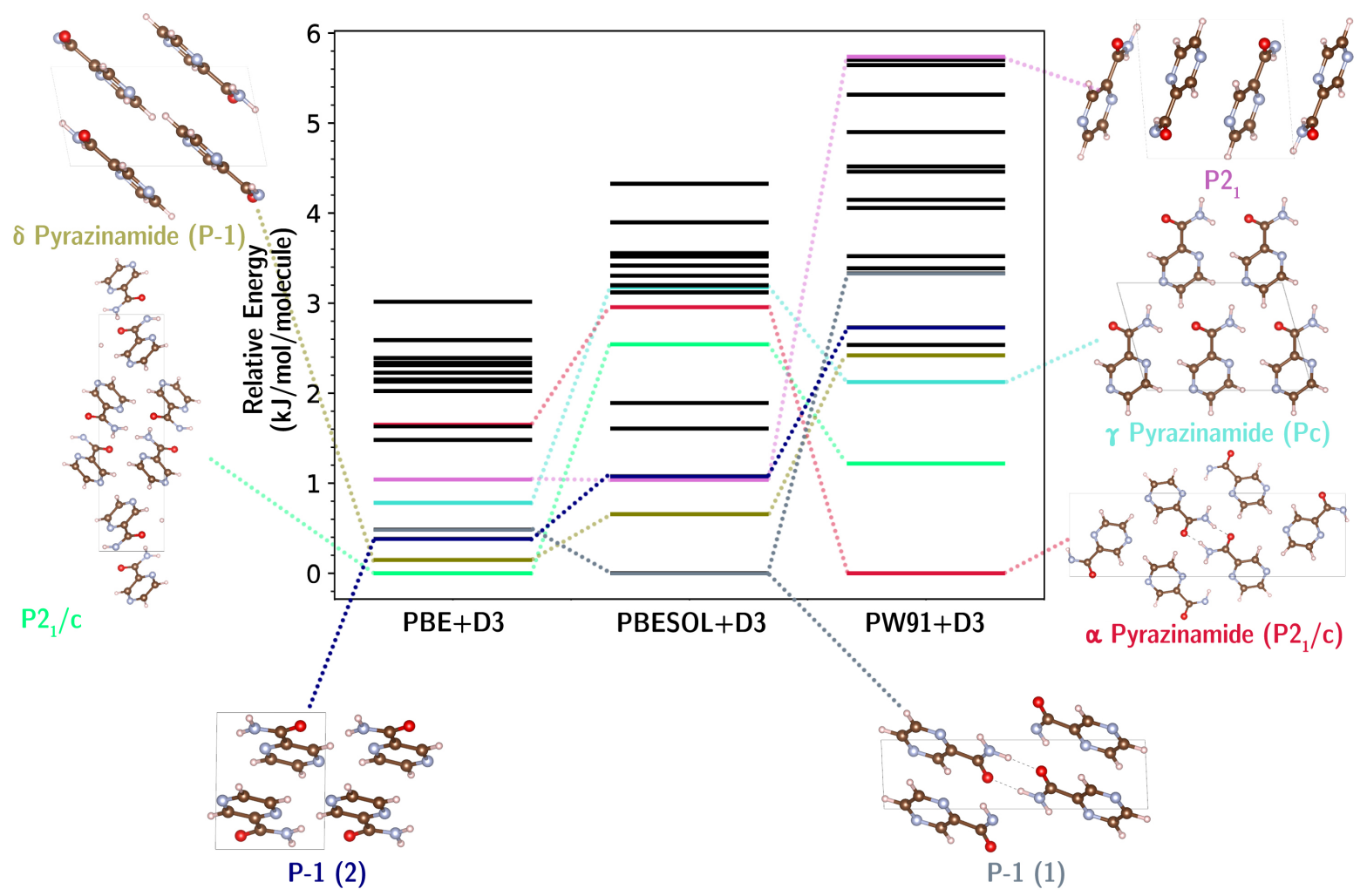

FIG. S7. Relative total energies for low energy pyrazinamide crystals obtained from different dispersion-inclusive DFT methods.
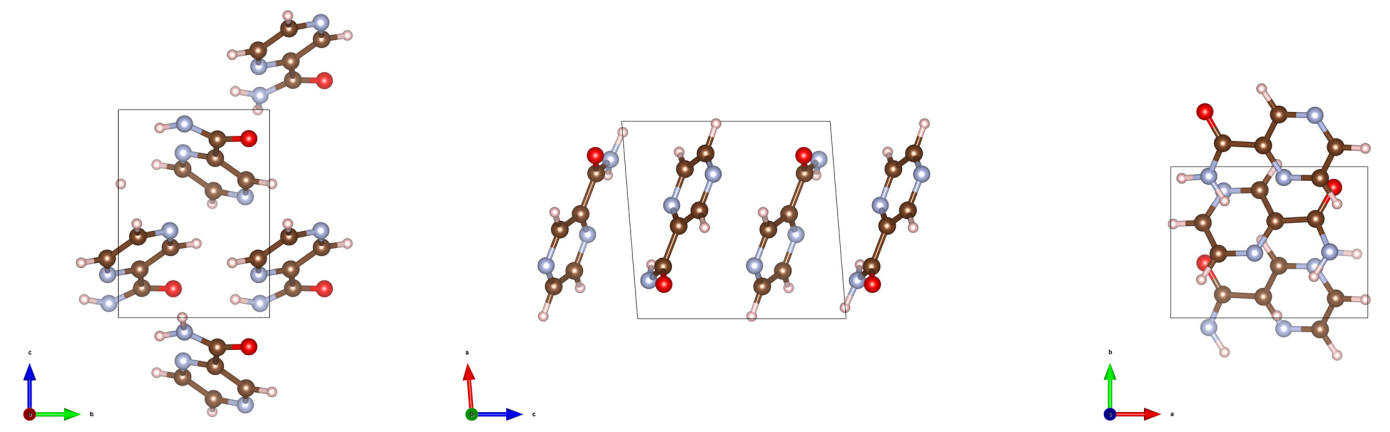

FIG. S8. Crystal structure of phonon stable monoclinic structure P2_1 of pyrazinamide. 


\begin{tabular}{|c|c|c|c|c|}
\hline Space group name & $\mathbf{a}[\AA]$ & $\mathbf{b}[\AA]$ & $\mathbf{c}[\AA]$ & Cell volume $\left[\AA^{3}\right]$ \\
\hline \multirow{3}{*}{ P2_1 (\#4) } & 6.8077 & 5.2211 & 7.1619 & 253.6973 \\
\hline & $\alpha$ & $\beta$ & $\gamma$ & \\
\hline & 90.0000 & 94.6431 & 90.0000 & \\
\hline Species & $\mathrm{x}$ & $\mathbf{y}$ & $\mathbf{z}$ & Site \\
\hline $\mathrm{H}$ & 0.0373 & 0.0163 & 0.1415 & $2 \mathrm{a}$ \\
\hline $\mathrm{H}$ & 0.2258 & 0.2737 & 0.4125 & $2 \mathrm{a}$ \\
\hline $\mathrm{H}$ & 0.3477 & 0.7532 & 0.7641 & $2 \mathrm{a}$ \\
\hline $\mathrm{H}$ & 0.4452 & 0.4225 & 0.5026 & $2 \mathrm{a}$ \\
\hline $\mathrm{H}$ & 0.4926 & 0.1211 & 0.9534 & $2 \mathrm{a}$ \\
\hline $\mathrm{C}$ & 0.0291 & 0.6406 & 0.2664 & $2 \mathrm{a}$ \\
\hline $\mathrm{C}$ & 0.0505 & 0.3455 & 0.8400 & $2 \mathrm{a}$ \\
\hline $\mathrm{C}$ & 0.2356 & 0.6550 & 0.3570 & $2 \mathrm{a}$ \\
\hline $\mathrm{C}$ & 0.2609 & 0.9245 & 0.7856 & $2 \mathrm{a}$ \\
\hline $\mathrm{C}$ & 0.3407 & 0.1296 & 0.8916 & $2 \mathrm{a}$ \\
\hline $\mathrm{N}$ & 0.0759 & 0.9283 & 0.7078 & $2 \mathrm{a}$ \\
\hline $\mathrm{N}$ & 0.2350 & 0.3402 & 0.9207 & $2 \mathrm{a}$ \\
\hline $\mathrm{N}$ & 0.3069 & 0.4369 & 0.4319 & $2 \mathrm{a}$ \\
\hline $\mathrm{O}$ & 0.3269 & 0.8637 & 0.3593 & $2 \mathrm{a}$ \\
\hline
\end{tabular}

TABLE S5. Crystallographic data the monoclinic structure P2_1 of pyrazinamide.


FIG. S9. Crystal structure of phonon stable monoclinic structure P2_1/c of pyrazinamide. 


\begin{tabular}{|c|c|c|c|c|}
\hline Space group name & $\mathbf{a}[\AA]$ & $\mathbf{b}[\AA]$ & $\mathbf{c}[\AA]$ & Cell volume $\left[\AA^{3}\right]$ \\
\hline \multirow[t]{3}{*}{ P2_1/c (\#14) } & 5.5027 & 5.1535 & 18.7785 & 532.0100 \\
\hline & $\alpha$ & $\beta$ & $\gamma$ & \\
\hline & 90.0000 & 92.5326 & 90.0000 & \\
\hline Species & $\mathbf{x}$ & $\mathbf{y}$ & $\mathbf{z}$ & Site \\
\hline $\mathrm{H}$ & 0.0109 & 0.7246 & 0.7709 & $4 \mathrm{e}$ \\
\hline $\mathrm{H}$ & 0.0796 & 0.7145 & 0.0145 & $4 \mathrm{e}$ \\
\hline $\mathrm{H}$ & 0.0823 & 0.5340 & 0.6310 & $4 \mathrm{e}$ \\
\hline $\mathrm{H}$ & 0.3140 & 0.5883 & 0.4811 & $4 \mathrm{e}$ \\
\hline $\mathrm{H}$ & 0.4203 & 0.2319 & 0.3235 & $4 \mathrm{e}$ \\
\hline $\mathrm{C}$ & 0.1445 & 0.1899 & 0.6959 & $4 \mathrm{e}$ \\
\hline $\mathrm{C}$ & 0.1951 & 0.1374 & 0.1411 & $4 \mathrm{e}$ \\
\hline $\mathrm{C}$ & 0.2781 & 0.5534 & 0.9355 & $4 \mathrm{e}$ \\
\hline $\mathrm{C}$ & 0.4667 & 0.5604 & 0.1672 & $4 \mathrm{e}$ \\
\hline $\mathrm{C}$ & 0.4850 & 0.6096 & 0.8884 & $4 \mathrm{e}$ \\
\hline $\mathrm{N}$ & 0.2230 & 0.7397 & 0.9812 & $4 \mathrm{e}$ \\
\hline $\mathrm{N}$ & 0.2815 & 0.5207 & 0.2096 & $4 \mathrm{e}$ \\
\hline $\mathrm{N}$ & 0.3807 & 0.1768 & 0.0988 & $4 \mathrm{e}$ \\
\hline $\mathrm{O}$ & 0.1687 & 0.1597 & 0.4300 & $4 e$ \\
\hline
\end{tabular}

TABLE S6. Crystallographic data the monoclinic structure P2_1/c of pyrazinamide.
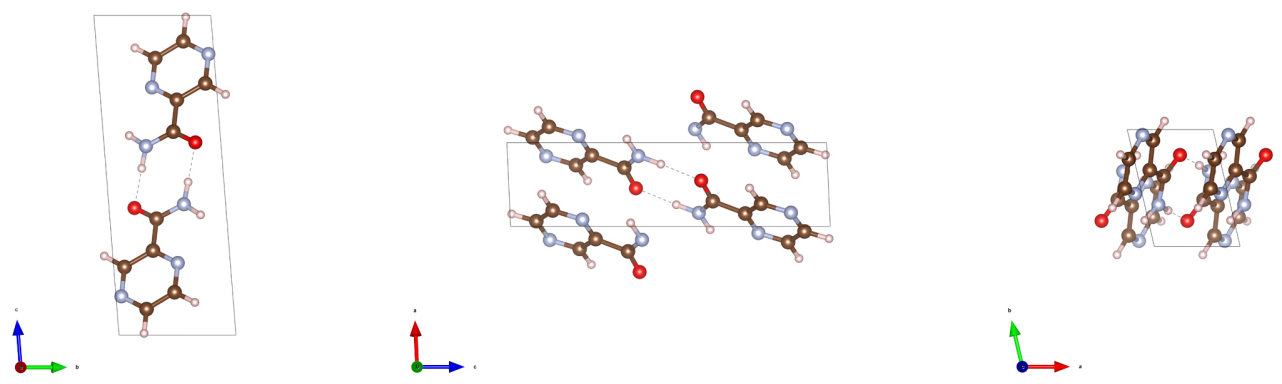

FIG. S10. Crystal structure of Pyrazinamide P-1(1). 


\begin{tabular}{|c|c|c|c|c|}
\hline Space group name & $\mathbf{a}[\AA]$ & $\mathbf{b}[\AA]$ & $\mathbf{c}[\AA]$ & Cell volume $\left[\AA^{3}\right]$ \\
\hline \multirow{3}{*}{$\mathrm{P}-1(\# 2)$} & 3.6364 & 5.0808 & 13.5700 & 243.5611 \\
\hline & $\alpha$ & $\beta$ & $\gamma$ & \\
\hline & 94.0438 & 91.5702 & 102.8816 & \\
\hline Species & $\mathrm{x}$ & $\mathbf{y}$ & $\mathbf{z}$ & Site \\
\hline $\mathrm{H}$ & 0.0406 & 0.7778 & 0.3759 & $2 \mathrm{i}$ \\
\hline $\mathrm{H}$ & 0.1470 & 0.2153 & 0.0053 & $2 \mathrm{i}$ \\
\hline $\mathrm{H}$ & 0.2576 & 0.3064 & 0.5215 & $2 \mathrm{i}$ \\
\hline $\mathrm{H}$ & 0.3840 & 0.6703 & 0.1021 & $2 \mathrm{i}$ \\
\hline $\mathrm{H}$ & 0.4565 & 0.0726 & 0.7527 & $2 \mathrm{i}$ \\
\hline $\mathrm{C}$ & 0.0614 & 0.2528 & 0.0811 & $2 \mathrm{i}$ \\
\hline $\mathrm{C}$ & 0.1530 & 0.6527 & 0.7357 & $2 \mathrm{i}$ \\
\hline $\mathrm{C}$ & 0.1886 & 0.5037 & 0.1339 & $2 \mathrm{i}$ \\
\hline $\mathrm{C}$ & 0.2696 & 0.9071 & 0.7870 & $2 \mathrm{i}$ \\
\hline $\mathrm{C}$ & 0.2997 & 0.6011 & 0.6361 & $2 \mathrm{i}$ \\
\hline $\mathrm{N}$ & 0.0808 & 0.5510 & 0.2254 & $2 \mathrm{i}$ \\
\hline $\mathrm{N}$ & 0.1605 & 0.3576 & 0.5907 & $2 \mathrm{i}$ \\
\hline $\mathrm{N}$ & 0.1634 & 0.9537 & 0.8789 & $2 \mathrm{i}$ \\
\hline $\mathrm{O}$ & 0.4541 & 0.2176 & 0.3973 & $2 \mathrm{i}$ \\
\hline
\end{tabular}

TABLE S7. Crystallographic data the triclinic structure P-1(1) of pyrazinamide.


FIG. S11. Crystal structure of Pyrazinamide P-1(2). 


\begin{tabular}{|c|c|c|c|c|}
\hline Space group name & $\mathbf{a}[\AA]$ & $\mathbf{b}[\AA]$ & $\mathbf{c}[\AA]$ & Cell volume $\left[\AA^{3}\right]$ \\
\hline \multirow{3}{*}{$\mathrm{P}-1(\# 2)$} & 5.5614 & 6.6946 & 6.8969 & 245.9160 \\
\hline & $\alpha$ & $\beta$ & $\gamma$ & \\
\hline & 89.0320 & 73.3098 & 89.1255 & \\
\hline Species & $\mathrm{x}$ & $\mathrm{y}$ & $\mathrm{z}$ & Site \\
\hline $\mathrm{H}$ & 0.0439 & 0.7559 & 0.4769 & $2 \mathrm{i}$ \\
\hline $\mathrm{H}$ & 0.2097 & 0.4556 & 0.9926 & $2 \mathrm{i}$ \\
\hline $\mathrm{H}$ & 0.2143 & 0.8501 & 0.7612 & $2 \mathrm{i}$ \\
\hline $\mathrm{H}$ & 0.2975 & 0.9579 & 0.9699 & $2 \mathrm{i}$ \\
\hline $\mathrm{H}$ & 0.3428 & 0.6358 & 0.20149 & $2 \mathrm{i}$ \\
\hline $\mathrm{C}$ & 0.1122 & 0.2742 & 0.5853 & $2 \mathrm{i}$ \\
\hline $\mathrm{C}$ & 0.2527 & 0.3883 & 0.8423 & $2 \mathrm{i}$ \\
\hline $\mathrm{C}$ & 0.3583 & 0.2297 & 0.4749 & $2 \mathrm{i}$ \\
\hline $\mathrm{C}$ & 0.4110 & 0.1423 & 0.2689 & $2 \mathrm{i}$ \\
\hline $\mathrm{C}$ & 0.4988 & 0.3402 & 0.7328 & $2 \mathrm{i}$ \\
\hline $\mathrm{N}$ & 0.0597 & 0.3560 & 0.7686 & $2 \mathrm{i}$ \\
\hline $\mathrm{N}$ & 0.3478 & 0.8833 & 0.8315 & $2 \mathrm{i}$ \\
\hline $\mathrm{N}$ & 0.4480 & 0.7369 & 0.4517 & $2 \mathrm{i}$ \\
\hline $\mathrm{O}$ & 0.2315 & 0.0989 & 0.2021 & $2 \mathrm{i}$ \\
\hline
\end{tabular}

TABLE S8. Crystallographic data the triclinic structure P-1(2) of pyrazinamide. 


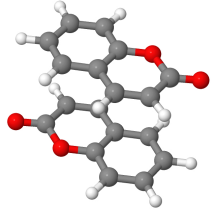

(a) Cou-1

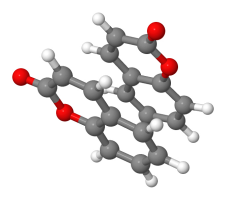

(d) Cou-4

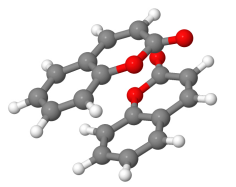

(g) Cou-7

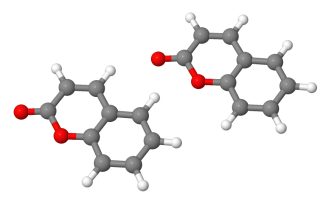

(j) Cou-10

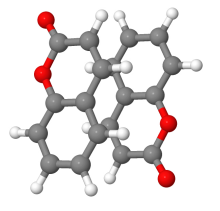

(b) Cou-2

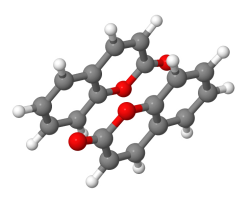

(e) Cou-5

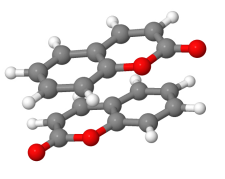

(h) Cou- 8

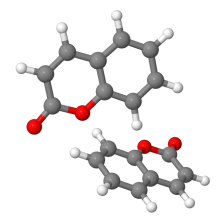

(k) Cou-11

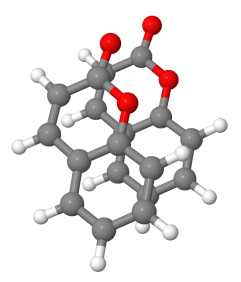

(m) Cou-13



(c) $\mathrm{Cou}-3$

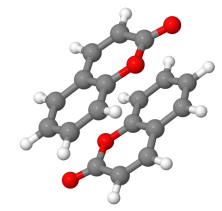

(f) Cou-6

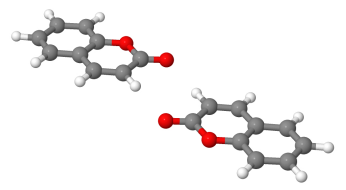

(i) Cou-9

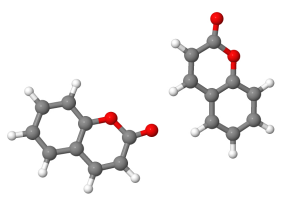

(l) Cou-12

FIG. S12. Coumarin dimers which used as identifier of dendrogram clusters 


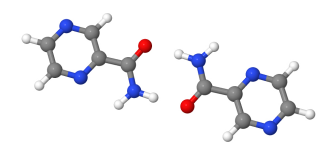

(a) Pyr-1



(d) Pyr-4

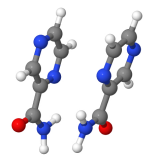

(g) Pyr-7



(j) Pyr-10



(m) Pyr-13

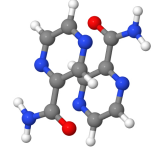

(b) Pyr-2

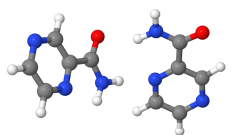

(e) Pyr-5

(f) Pyr-6

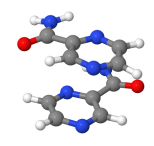

(h) Pyr-8

(i) Pyr-9



(k) Pyr-11

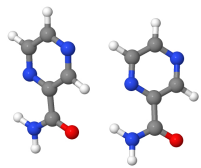

(l) Pyr-12

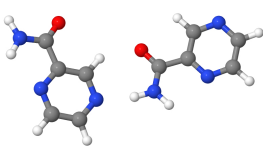

FIG. S13. Pyrazinamide dimers which used as identifier of dendrogram clusters 


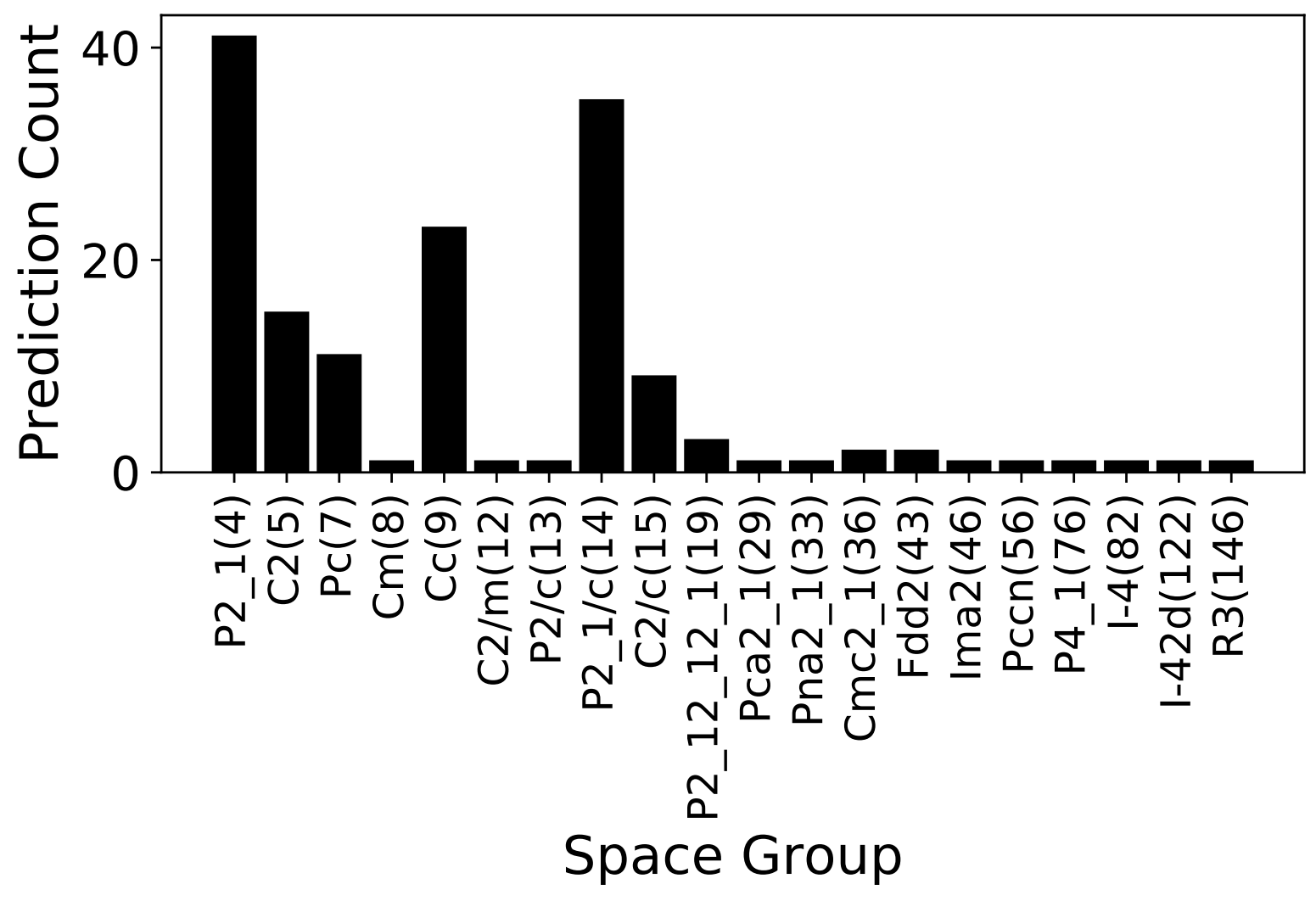

FIG. S14. Space group distrubution of DFT relaxed cytosine structures. (P1 and P-1 are excluded) 


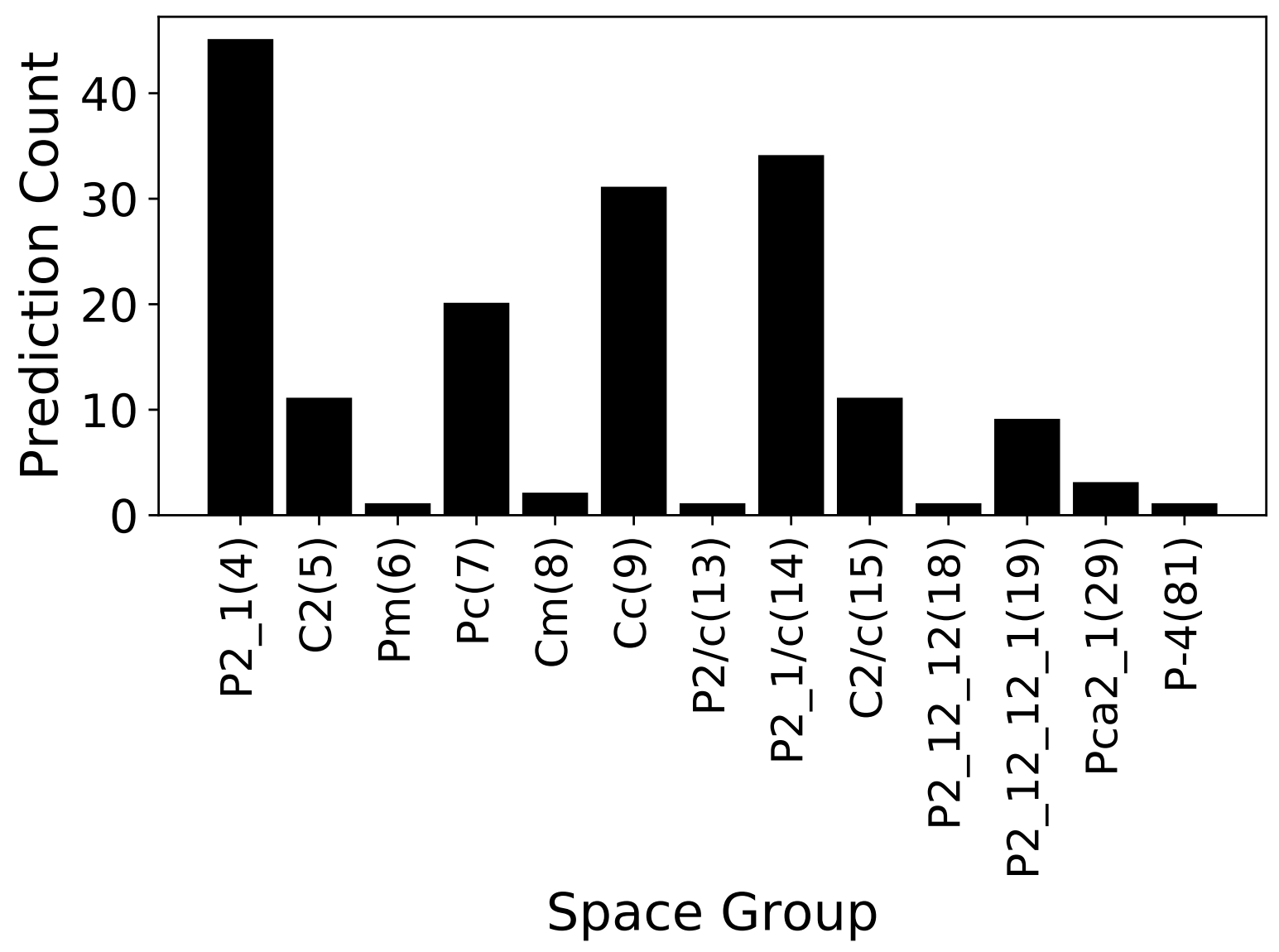

FIG. S15. Space group distrubution of DFT relaxed coumarin structures. (P1 and P-1 are excluded) 


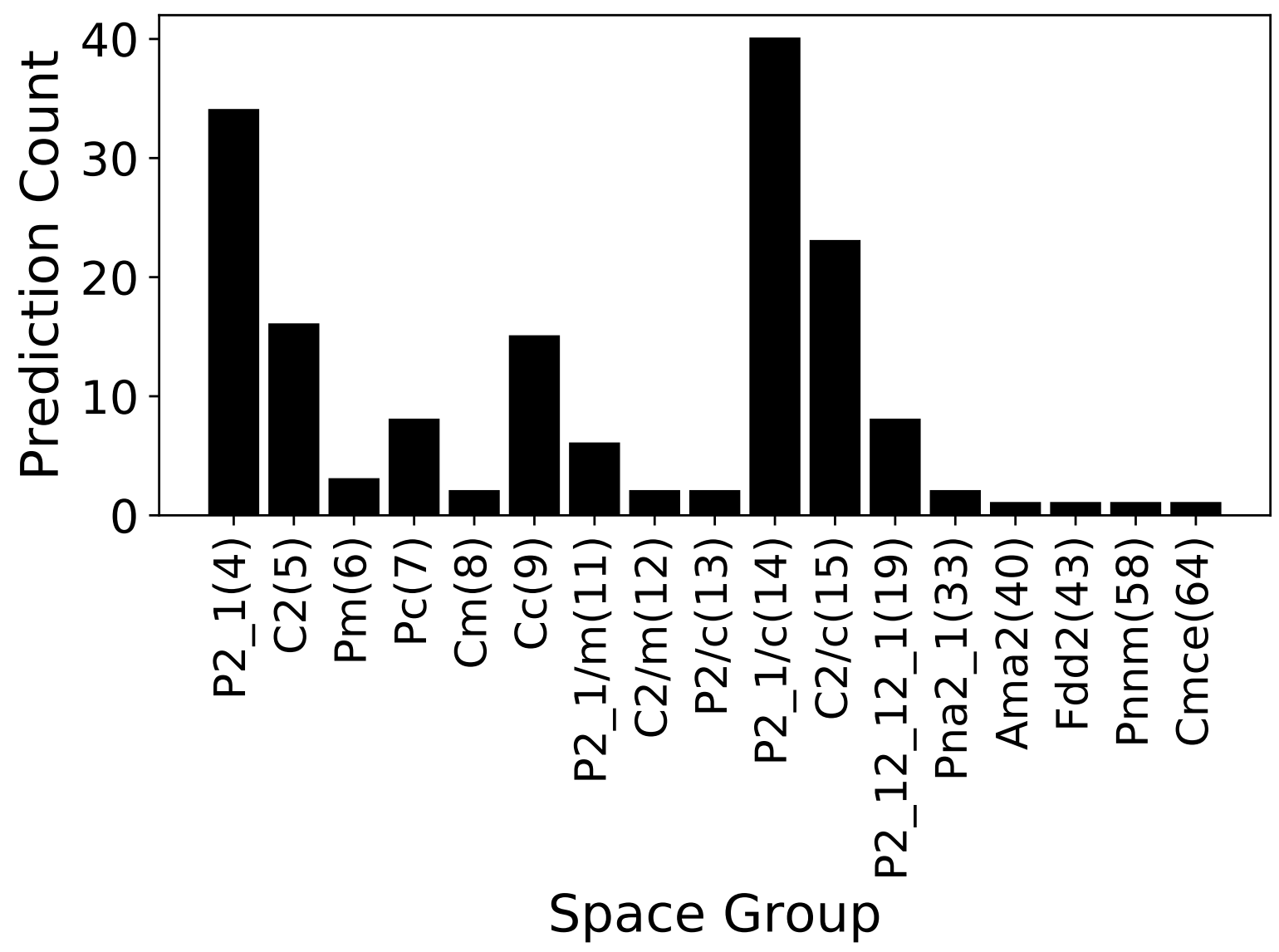

FIG. S16. Space group distrubution of DFT relaxed pyrazinamide structures. (P1 and P-1 are excluded) 